Classification

Physics Abstracts

$61.40-07.50-72.20 \mathrm{P}$

\title{
Appareillage automatisé de mesure simultanée du pouvoir thermoélectrique et de la conductivité électrique. Application à l'étude de couches polymères semi-conductrices
}

\author{
A. Moliton ( ${ }^{1}$ ), B. Ratier ( $\left.{ }^{1}\right)$, C. Moreau ( ${ }^{1}$ ) et G. Froyer $\left({ }^{2}\right)$ \\ (1) LEPOFI, Faculté des Sciences, 87060 Limoges Cedex, France \\ (2) Lab. OCM, CNET, 22301 Lannion Cedex; France
}

(Reģu le 19 novembre 1990, accepté le 22 janvier 1991)

\begin{abstract}
Résumé. - Nous présentons dans cet article un système de mesure simultanée de la conductivité $\sigma$ et du pouvoir thermoélectrique $S$ : il permet des mesures en fonction de la température (entre $130 \mathrm{~K}$ et $360 \mathrm{~K}$ ) dans le cas de couches semi-conductrices relativement fragiles. A titre d'application, nous indiquons les résultats que nous avons obtenus dans le cas de couches polymères (PPP) implantées avec des ions sodium : alors que seule une semi-conduction par défaut est générée par de fortes énergies d'implantation $(E=250 \mathrm{keV})$, il apparaît une semiconduction induite par le dopage $\mathrm{n}$ lors d'implantations à basse énergie $(E=30 \mathrm{keV})$.

Abstract. - In this paper, we present an automatized system for simultaneous measurement of conductivity $\sigma$ and thermoelectric power $S$ : measurements are allowed for temperatures ranging from $130 \mathrm{~K}$ to $360 \mathrm{~K}$ on brittle semiconductor layers. As an example of the application, results obtained in the case of polymer (PPP) layers implanted with $\mathrm{Na}$ ions are presented : with high energy implantation $(E=250 \mathrm{keV})$ we observe only a defect semiconduction of $\mathrm{p}$ type while at low energy $(30 \mathrm{keV})$ an electronic $\mathrm{n}$ type conduction appears.
\end{abstract}

\section{Introduction : cadre de l'étude.}

L'analyse des mécanismes de transport dans les matériaux nécessite la connaissance simultanée de la conductivité électrique continue $(\sigma)$ et du pouvoir $(S)$ en fonction de la température $T$. Une évolution linéaire de $\log \sigma$ et de $S$ en fonction de l'inverse de la température est caractéristique de phénomènes thermiquement activés, alors qu'une évolution linéaire de $\log \sigma \sqrt{T}$ et de $S / T$ en fonction de $T^{-1 / 4}$ correspond à des mécanismes de sauts de porteurs à distance variable.

Pour notre part, nous avons étudié de tels phénomènes dans le cas de couches implantées de polymères électroactifs (polyparaphénylène noté PPP, principalement) : selon les paramètres d'implantation [1], la couche finalement obtenue est soit conductrice (fortes énergies), soit semi-conductrice. Dans ce dernier cas, les lois d'évolution de $\sigma$ et de $S$ en fonction de la 
température ont été étudiées [2] dans le cadre du modèle de bande des semi-conducteurs amorphes. Ce modèle est effectivement une approche alternative à celle provenant de l'étude plus microscopique (chaîne isolée) qui conduit [3] cependant à l'introduction de niveaux localisés au-dessus de la bande de valence ou au-dessous de la bande de conduction (bandes de polarons ou de bipolarons): dans la représentation des semi-conducteurs amorphes, ce sont des niveaux localisés dans les queues de bande qui se situent à ces énergies, alors qu'au voisinage de $E_{\mathrm{F}}$ sont introduits des niveaux localisés attribuables à des défauts (liaisons pendantes par exemple).

Dans le cadre de ce modèle [4], et aux plus basses températures, le mécanisme de saut à distance variable (entre ces niveaux localisés dus à des défauts) est en général prépondérant ; lorsque la température croît, les lois d'évolution de $\sigma$ et de $S$ en fonction des mécanismes de transport dans les différents niveaux d'énergie sont caractérisées par des énergies d'activation d'autant plus grandes que la température est élevée. De plus, et ainsi que le souligne Mott [5], le signe de $S$ est un indicateur fiable du type de semi-conduction n ou p du matériau (ce qui n'est pas forcément le cas du coefficient Hall).

Cependant, ainsi qu'on va le voir au paragraphe suivant, il existe un problème majeur : celui de la réalisation des mesures, notamment en fonction de la température. Jusqu'alors, seuls ont été développés des appareillages destinés à effectuer pour le pouvoir thermoélectrique soit des mesures volumiques $[6,7,8]$, soit des mesures de surface [9]. Ce dernier appareillage, limité en plage de température (température maximum de fonctionnement voisine de $330 \mathrm{~K}$ ) et à la seule mesure du pouvoir thermoélectrique, nous a permis de percevoir l'amorce d'un comportement spécifique pour un polymère électroactif (PPP) implanté à basse énergie. C'est pour étudier, au mieux et de façon systématique, cet important phénomène que nous nous sommes attachés à réaliser un banc automatisé capable d'effectuer la mesure sur des couches semi-conductrices de la conductivité, en plus de celle du pouvoir thermoélectrique, et ce sur un plus large domaine de température (130 K à $360 \mathrm{~K})$.

\section{Montage expérimental.}

Outre les difficultés générales de la mesure de $S$ en fonction de $T$, provenant (Mott [5]) de la mesure de très faibles tensions à travers des matériaux très résistifs, nous avons dû résoudre des difficultés particulières liées :

- à des mesures délicates sur les couches implantées qu'il ne faut pas détériorer, tout en assurant un bon contact (avec les pointes de mesure) à toutes les températures,

- à l'existence d'une tension résiduelle (variable avec la température), présente même en l'absence de gradient de température entre les deux pointes, qui se superpose au potentiel thermoélectrique. Dans l'étude des systèmes vitreux, une telle difficulté expérimentale a été également rencontrée par Nagels [10]. Nous avons, pour notre part, constaté que cette tension résiduelle était généralement d'autant plus élevée que l'échantillon était porté à une température plus basse, donc plus résistif; l'origine de cette perturbation semble donc être imputable à la mauvaise qualité des contacts liés à l'étude de couches particulièrement résistives. A chaque mesure nous avons relevé systématiquement cette "tension d'offset $O$ " (en fait assimilable à une d.d.p. de contact) préalablement à l'établissement du gradient de température (que nous avons fixé à $9 \mathrm{~K}$ ) et, pour obtenir des valeurs correctes de $S$, nous avons déduit cette valeur $O$ de la tension finalement mesurée : cette tension $O$ variant en fonction de la température $T$, il est particulièrement important d'en tenir compte lors de l'étude de $S=f(T)$.

Pour effectuer de telles mesures, nous avons réalisé un banc fonctionnant entre $130 \mathrm{~K}$ et $360 \mathrm{~K}$ : les échantillons sont placés dans une enceinte maintenue sous vide primaire, afin 
d'éviter le "givrage 》 de ceux-ci lors du refroidissement par conduction thermique du porteéchantillon relié à une tige en cuivre plongée dans l'azote liquide. La remontée en température s'effectue à l'aide d'un thermocoax chauffant. Le banc est entièrement piloté par un HP 9825 et un bus IEEE (Fig. 1a et Fig. 1b). Les figures 2, 3, 4a et la nomenclature (Fig. 4b) qui suit, décrivent de façon détaillée l'enceinte de test.

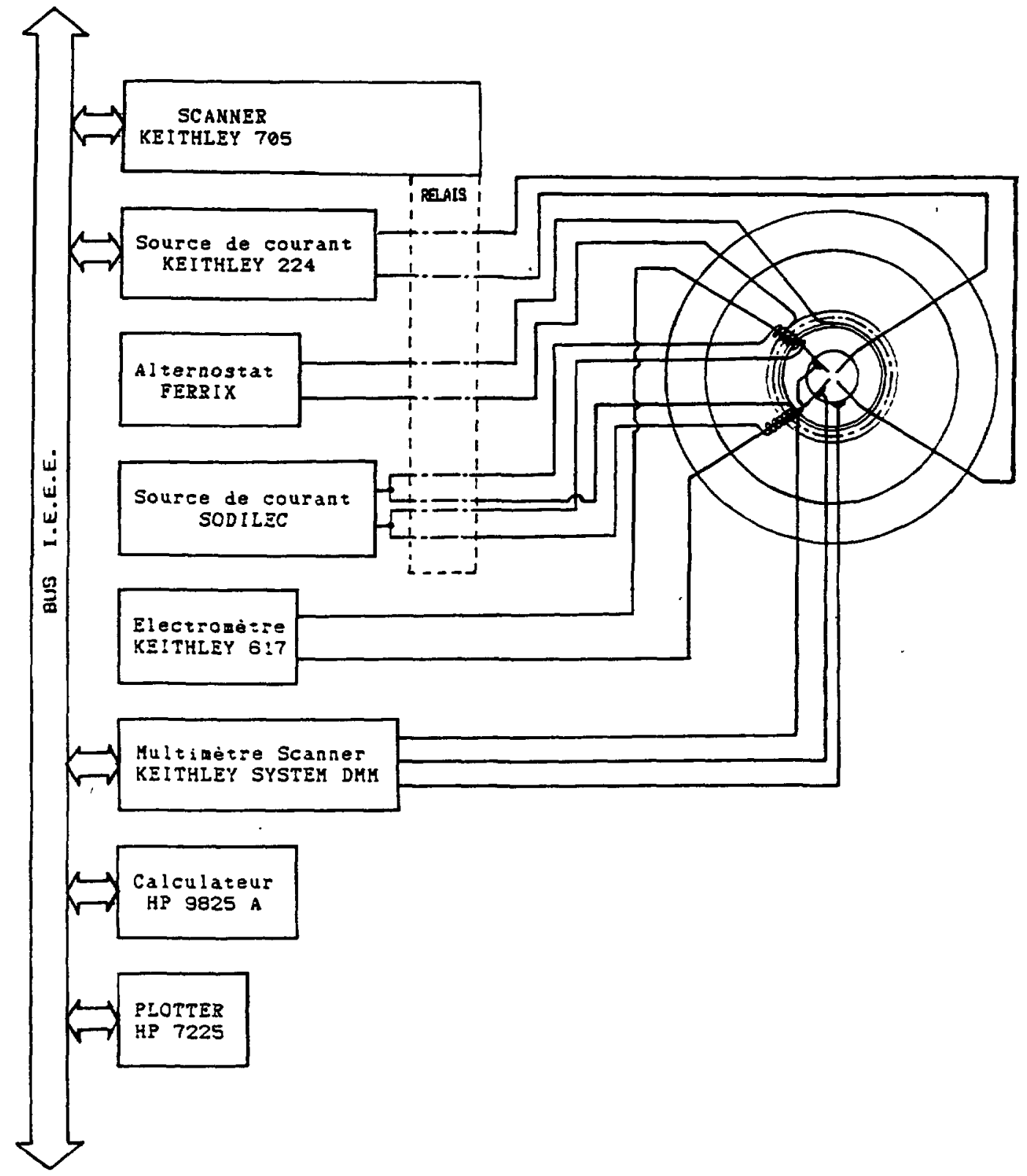

Fig. 1a. - Présentation générale du banc de mesure.

[General presentation of testing bench.] 


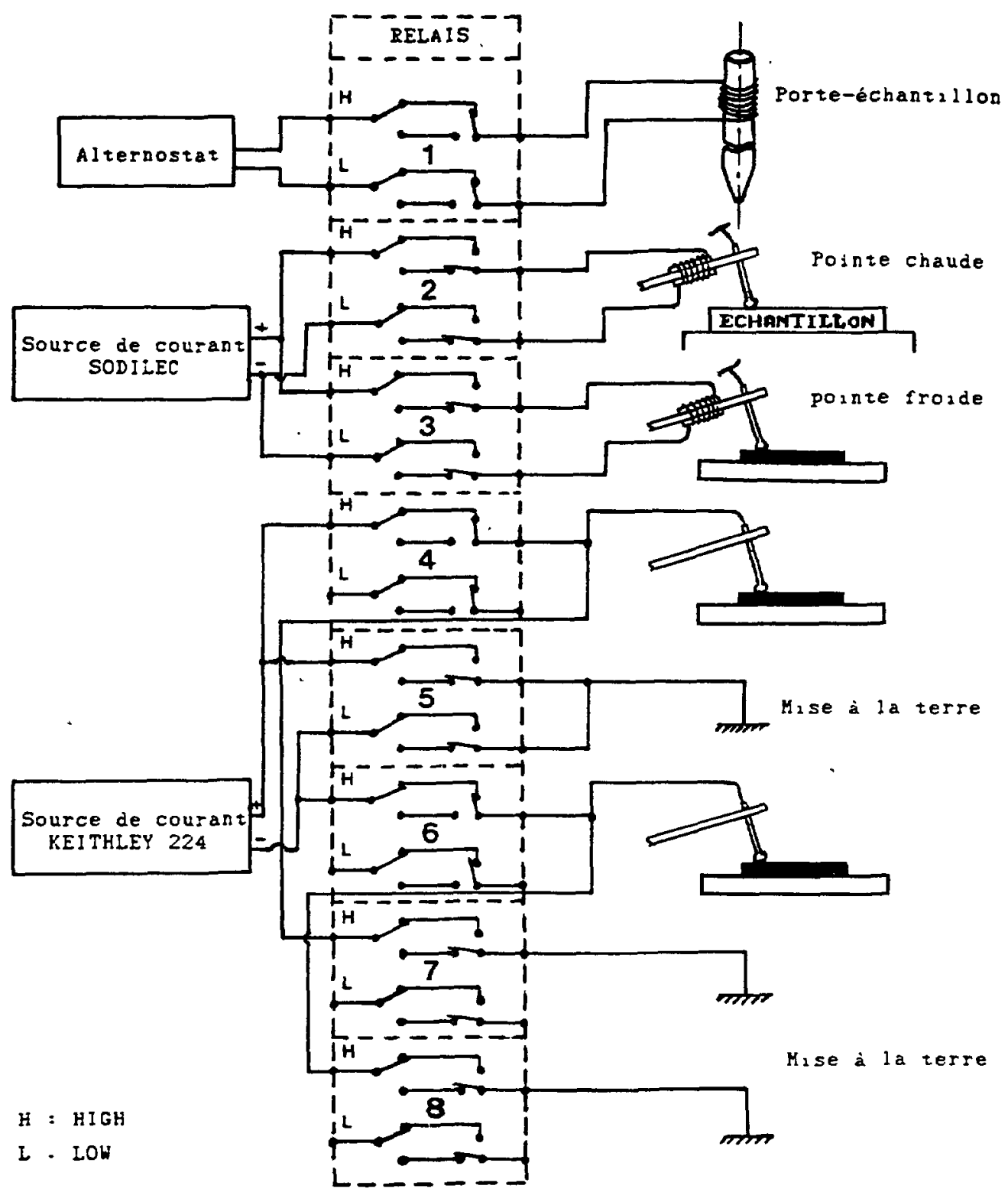

Fig. 1b. - Positionnement des relais du scanner Keithley 705.

[Relay configuration of Keithley 705 scanner.]

2.1 ELECTRODES DE TEST. - Les quatre pointes de test sont réalisées à partir de pointes de Karl Süss en tungstène, dont les extrémités ont été rendues sphériques pour éviter de détériorer les couches conductrices des échantillons. La formation de ces sphères a été réalisée au moyen d'une torche à plasma.

Deux de ces électrodes de tungstène sont connectées aux câbles coaxiaux de l'électromètre de mesure de la conductivité et du coefficient Seebeck, situés à l'extérieur de l'enceinte, par l'intermédiaire de fils de tungstène, dont les deux extrémités sont reliées par épissure à 


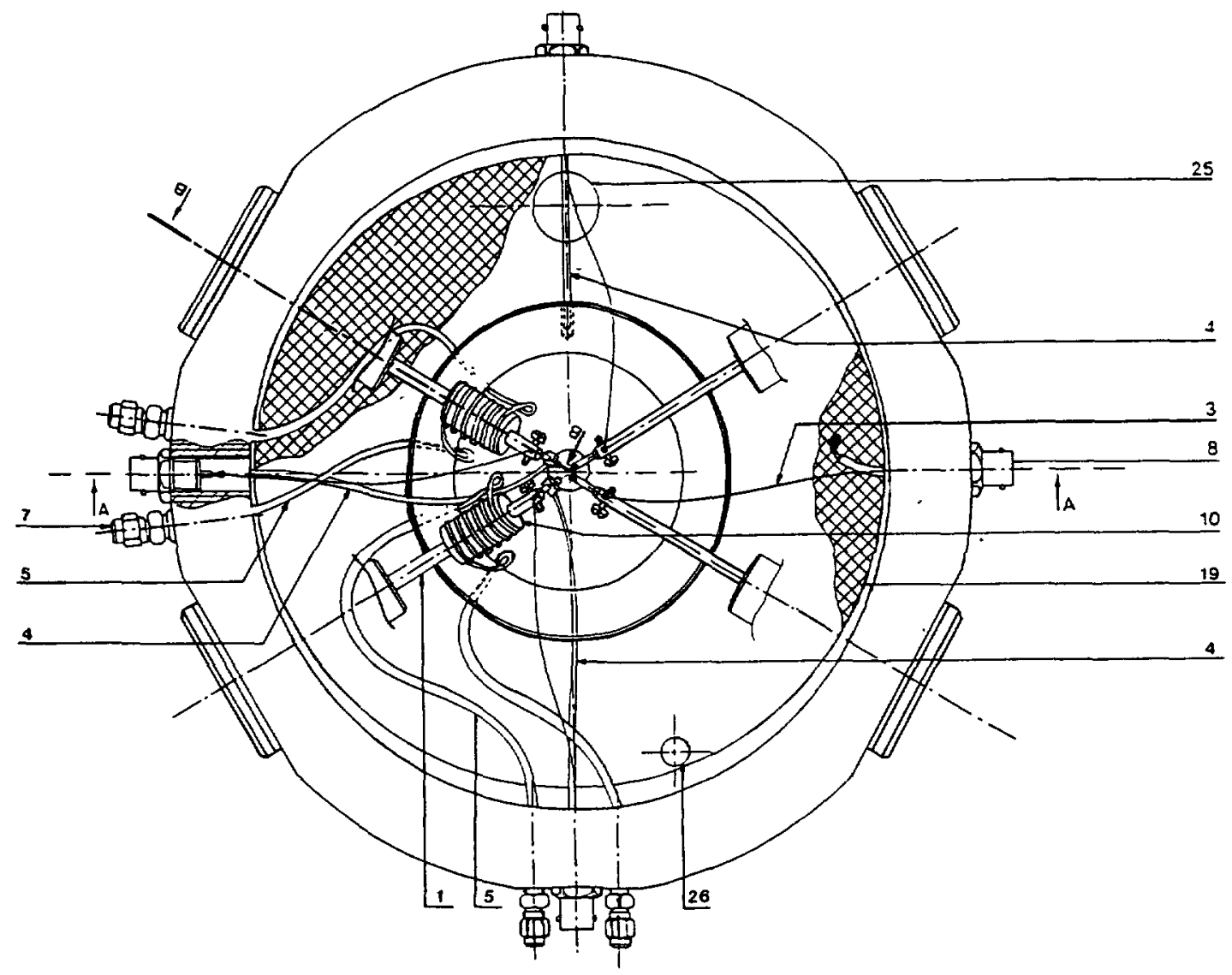

Fig. 2. - Vue de dessus du banc de mesure.

[Top view of testing bench.]

l'électrode et aux passages étanchès BNC: l'épissure (constituée par l'enroulement du fil autour de l'électrode ou de la cosse de connection du passage BNC) permet d'éviter l'utilisation de soudure. On a ainsi réalisé toute la connectique de l'enceinte de test avec un seul métal afin d'éviter toute création parasite de chaleur ou de différence de potentiel, dues à l'effet Peltier dans le cas de jonction de métaux différents.

Pour maintenir un. bon contact ohmique entre la couche implantée des échantillons et les électrodes, quelle que soit la température, nous avons mis au point un système de réglage par micromanipulateurs commandant la pression des pointes par l'intermédiaire de passages étanches de type "soufflet » (Fig. 3) : il est ainsi possible de rétablir, et ce quelle que soit la température de l'échantillon, le contact approprié entre les pointes et l'échantillon à l'aide des micromanipulateurs fixés à l'extérieur de l'enceinte de mesure.

Les deux autres pointes sont également réglables de l'extérieur avec un système identique de micromanipulateur agissant sur un soufflet auquel les pointes sont reliées mécaniquement mais isolées électriquement. Elles sont reliées également par câble coaxial et à travers des passages étanches à la source de courant. 


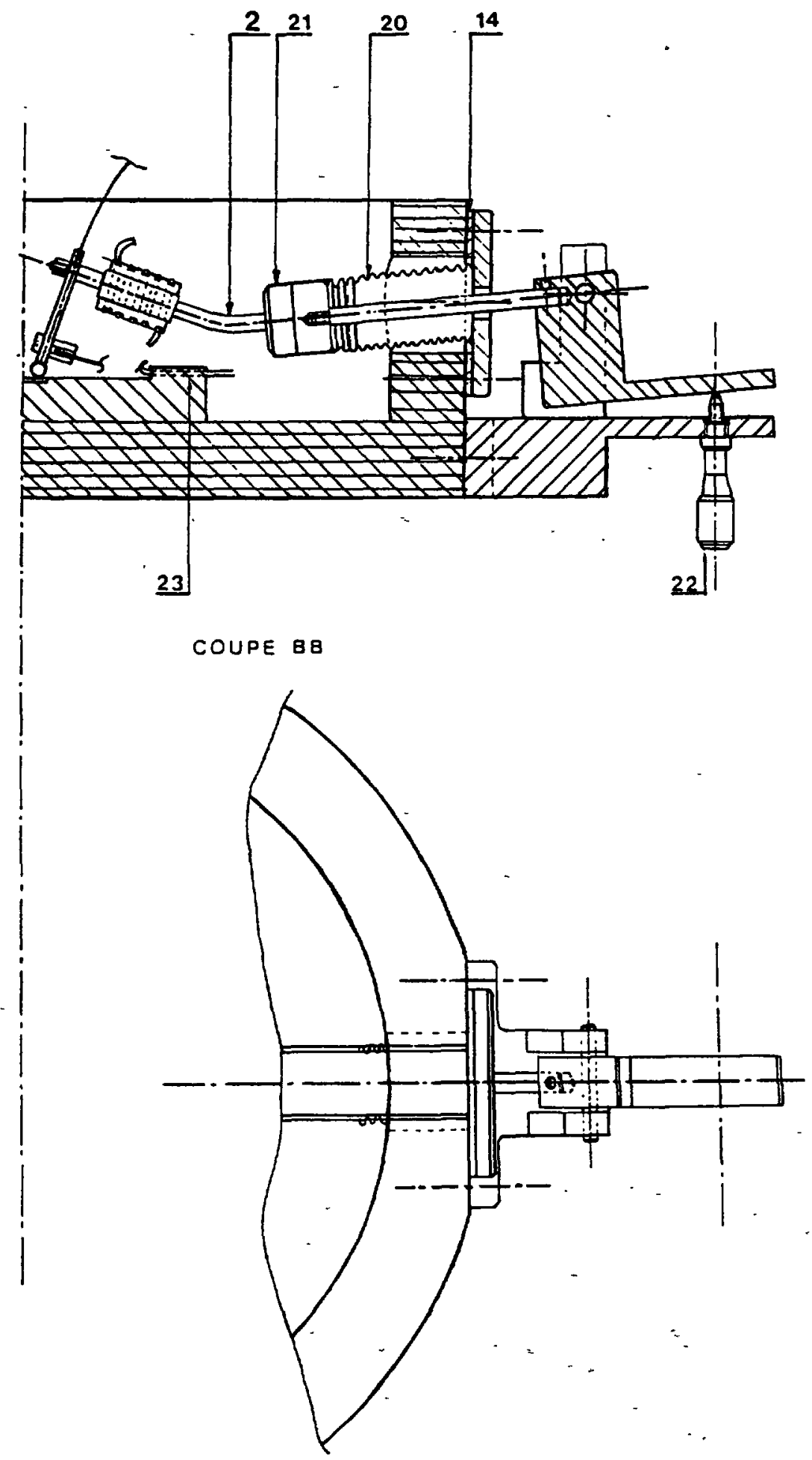

Fig. 3. - Système de réglage par micromanipulateurs.

[System of adjustment by micromanipulators.] 


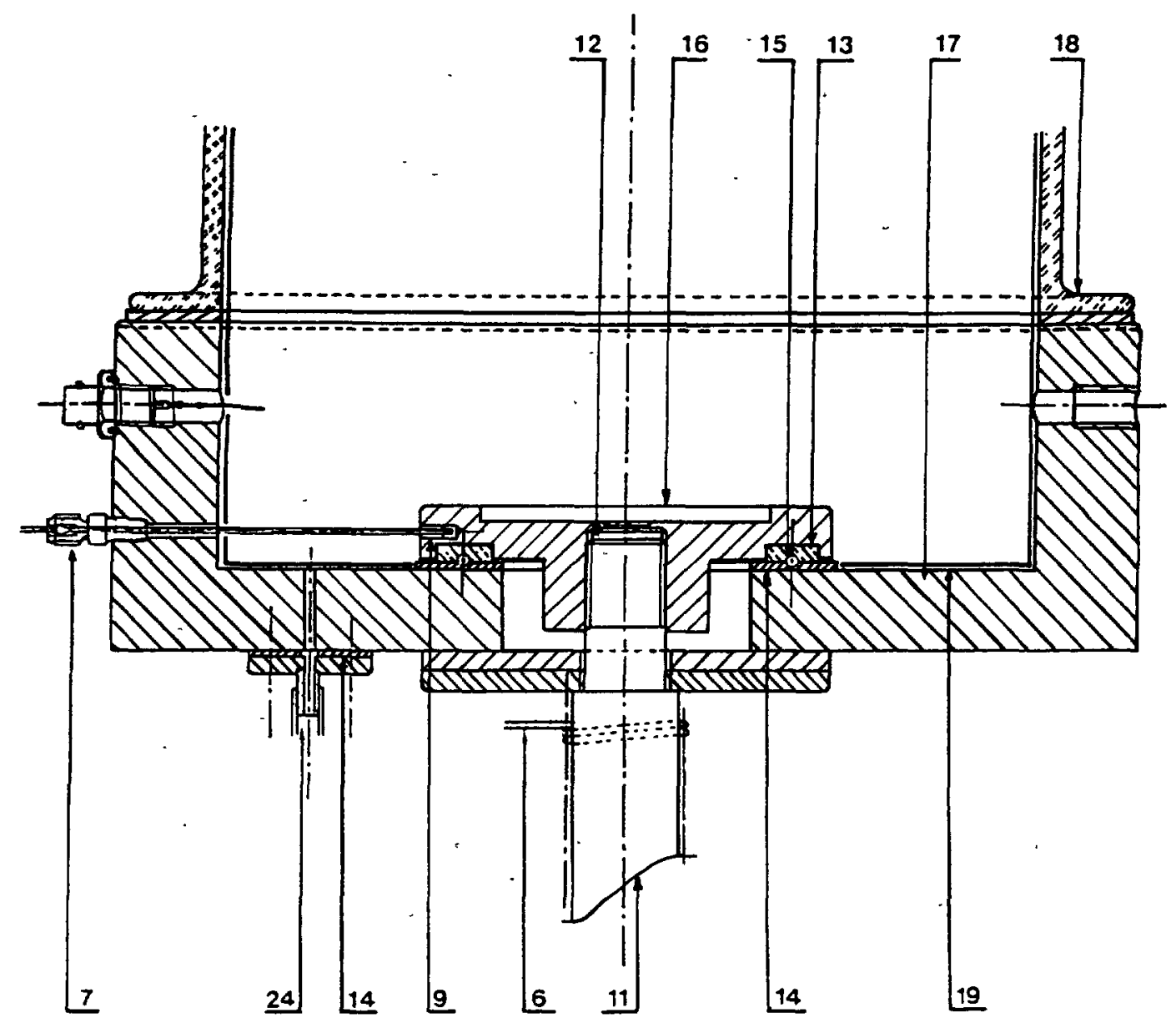

COUPE AA

Fig. 4a. - Vue en coupe du banc expérimental.

[Cross-section of experimental bench.]

2.2 MESURE DE $S$. - Le chauffage des deux pointes, servant à la mesure de la d.d.p., est effectué par des thermocoax blindés, isolés électriquement de celles-ci-par une pièce en nitrure de bore (conducteur thermique, isolant électrique), afin d'éviter les perturbations. La mesure de la température entre les pointes est réalisée à l'aide de thermocouples (de type thermocoax blindés également) introduits chacun dans un petit bloc en nitrure de bore fixé au plus près de l'extrémité de chaque pointe (Fig. 3).

L'évolution du coefficient thermoélectrique étant proportionnelle à l'écart de température entre pointes, le programme d'automatisation effectue deux mesures pour tenir compte des tensions d'offset :

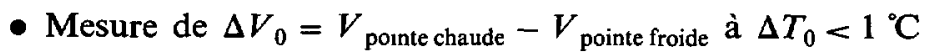

- Mesure de $\Delta V_{1}=V_{\text {pointe chaude }}-V_{\text {pointe froide }}$ à $\Delta T_{1}=9{ }^{\circ} \mathrm{C}$. 


\begin{tabular}{|c|c|c|c|c|}
\hline 26 & . & Passage de remise à l'air & & \\
\hline 25 & & Passage de pompage & & \\
\hline 24 & & Passage pour jauge & & \\
\hline 23 & & Passage de regulation thermique & & \\
\hline 22 & 4 & $\begin{array}{l}\text { Bubée ricotmérrique de pression } \\
\text { contuct }\end{array}$ & & Microcontrol \\
\hline 21 & 4 & Isolent therraique de pointe de lest & Tétlon & colle \\
\hline 20 & 4 & Passage étanche de manipulation & & "Tombac" \\
\hline 19 & 1 & Cage de Faraday & & Grille \\
\hline 18 & 1 & Cloche à vide & Verre & \\
\hline 17 & 1 & Bati isolant thermique & Terion & \\
\hline 16 & 1 & Porte-échanbillon & $\mathrm{Z8C17}$ & Inoxydable \\
\hline 15 & 1 & Joint torique d'étanchéite & & \\
\hline 14 & 8 & Joint plat d'étanchéité & & \\
\hline 13 & 1 & Isolant therumque & Terion & \\
\hline 12 & 1 & Poudre "contact thermique" & $\begin{array}{l}\text { Niture } \\
\text { de bore }\end{array}$ & En poudre \\
\hline 11 & 1 & Pointe refoidissement/chauffage ech. & $\mathrm{Cu} \mathrm{Pb} 1$ & \\
\hline 10 & 2 & Corps de four (Isolant electrique) & $\begin{array}{l}\text { Niture } \\
\text { de bore }\end{array}$ & \\
\hline 9 & 5 & Entretoise d'isolement des thermocouples & Ietion & \\
\hline 8 & 4 & Passage eanche pour cable BNC & & Radiail \\
\hline 7 & 5 & Passage elanche Therrolok MG 10 & & Thermocoax \\
\hline 6 & 1 & Elément chauffant (I. éch.) ZZZZ 1-20 & & Thermocoax \\
\hline 5 & 1 & Elément chauffant pointe chaude $1 \mathrm{~N} \mathrm{Cl} 10$ & & Thermocoax \\
\hline 4 & 3 & Themocouple SK2/NE & & Thermocoax \\
\hline 3 & 4 & Fil de Tungstène & $\mathbf{w}$ & \\
\hline 2 & 1 & Pointe froide & $\mathbf{W}$ & \\
\hline 1 & 1 & Pointe chaude & a & \\
\hline REP & NB & DESIGNATION & MATIERE & OBS \\
\hline \multicolumn{2}{|c|}{$\underset{1}{\text { ECIAELLE }}$} & \multicolumn{3}{|c|}{$\begin{array}{l}\text { BANC DE MESURE DU POUVOIR } \\
\text { THERMOELECTRIQUE ET DE LA CONDUCTIVITE }\end{array}$} \\
\hline & & LEPOFI & . & \\
\hline
\end{tabular}

Fig. 4b. - Nomenclature générale.

[General nomenclature.] 
Le coefficient Seebeck est finalement donné par :

$$
S=-\frac{\Delta V}{\Delta T}=-\frac{\Delta V_{1}-\Delta V_{0}}{\Delta T_{1}-\Delta T_{0}}
$$

Bien que cette méthode soit quelque peu critiquable (notamment par l'utilisation d'un gradient thermique relativement élevé qui suppose une loi d'évolution linéaire de $S$ en fonction de ce dernier, ce qui est cependant sensiblement vérifié), il est pratiquement impossible de manipuler différemment : l'utilisation d'un gradient thermique plus faible ne produirait qu'une faible variation de la tension qui est bien souvent de l'ordre de grandeur du bruit de la mesure.

En réalité, le schéma de mesure électrique peut être représenté sur la figure 5.

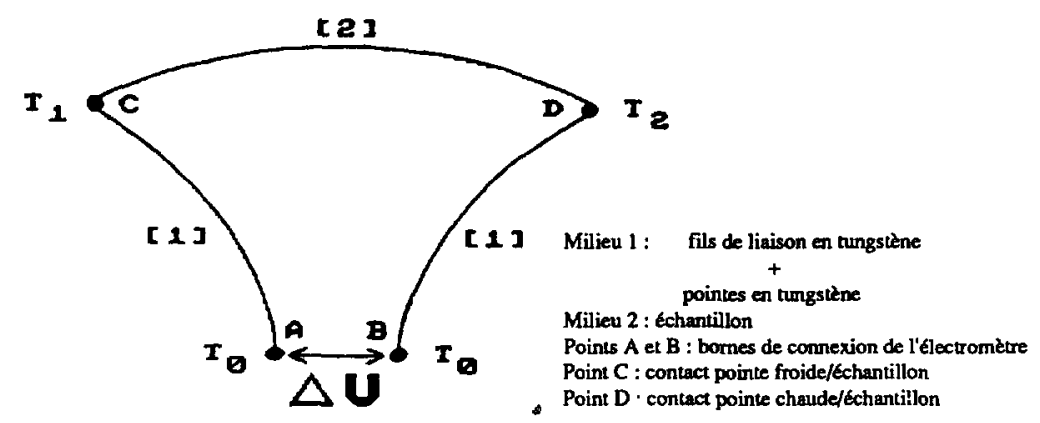

Fig. 5. - Schéma de mesure électrique.

[Electric diagram of measure.]

Si on appelle respectivement $\alpha_{1}$ et $\alpha_{2}$ les pouvoirs thermoélectriques absolus de l'échantillon et des fils en tungstène, on peut écrire en négligeant les différences de potentiels de contact :

$$
\Delta V=-\left(\alpha_{1}-\alpha_{2}\right) \Delta T
$$

Pour déterminer $\alpha_{1}$ en fonction de la température dans le cas du tungstène (que nous noterons $\alpha_{\mathrm{W}}$ ) nous avons mis comme échantillon une plaquette de cuivre dont le pouvoir thermoélectrique absolu $\alpha_{\mathrm{Cu}}$ en fonction de la température a été déterminé par ailleurs [11].

A partir des mesures de $-\frac{\Delta V}{\Delta T}$, on en a déduit la courbe $\alpha_{\mathrm{w}}=f(T)$ (Fig. 6) : la valeur parasite introduite par les fils de liaison, relativement faible $(\approx 6 \mu \mathrm{V} / \mathrm{K})$ et particulièrement constante en fonction de la température, ne modifie donc pas sensiblement les profils des courbes obtenues directement sans correction à partir de la formule précédente : $S=-\frac{\Delta V}{\Delta T}$

Finalement, le pouvoir thermoélectrique absolu $\alpha$ des échantillons étudiés est tel que : $\Delta V=-\left(\alpha-\alpha_{\mathrm{W}}\right) \Delta T, \quad$ soit $\quad S=-\frac{\Delta V}{\Delta T}=\alpha-\alpha_{\mathrm{W}}, \quad$ c'est-à-dire $\quad \alpha=S+\alpha_{\mathrm{W}}, \quad$ avec $\alpha_{\mathrm{W}} \approx 6 \mu \mathrm{V} / \mathrm{K}$.

2.3 Mesure de la conductivité $(\sigma)$. - Pour mesurer la conductivité, on utilise les deux pointes supplémentaires de même nature que les précédentes mais non munies de système de chauffage et de mesure de température. Elles servent à l'injection du courant de mesure et 


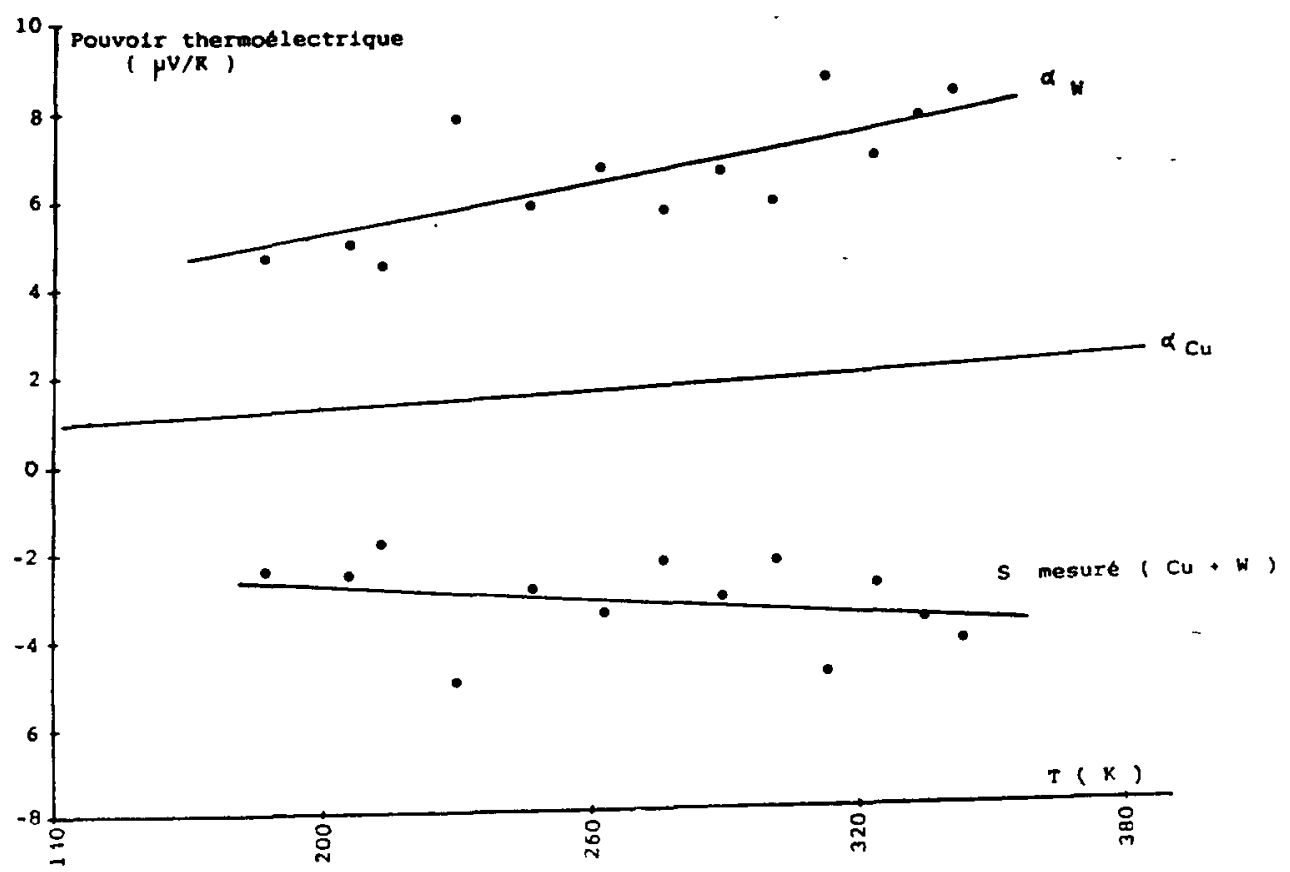

Fig. 6. - Pouvoir thermoélectrique absolu du tungstène en fonction de la température.

[Tungsten absolute thermoelectric power versus on temperature.]

forment une configuration carrée (de côté égal à $5 \mathrm{~mm}$ pour notre montage) avec les deux pointes de mesure de tension.

Dans ces conditions, la formule employée pour déterminer la conductivité d'une couche mince (épaisseur de la couche implantée comprise entre $60 \mathrm{~nm}$ pour des ions $\mathrm{Na}^{+}$de $30 \mathrm{keV}$ et $573 \mathrm{~nm}$ pour des ions $\mathrm{Na}^{+}$de $250 \mathrm{keV}$ : cf. Réfs. [1] ou [2]), a la forme habituelle [12] :

$$
\sigma=\frac{0,11}{e} \frac{l}{V}
$$

avec :

$$
\begin{aligned}
& e \text { en } \mathrm{cm}: \text { épaisseur de la couche conductrice } \\
& \frac{I}{V} \text { en } \Omega^{-1} \\
& \sigma \text { en } \Omega^{-1} \mathrm{~cm}^{-1}
\end{aligned}
$$

Une remarque expérimentalement importante doit être faite : lors de la mesure de la d.d.p. thermoélectrique (mesure de $S$ ) ces deux pointes doivent être reliées à la masse (Fig. 1b) afin de supprimer des perturbations électrostatiques qui seraient liées à un phénomène d'influence du milieu extérieur et transmises à l'enceinte de mesure (blindée par une cage de Faraday) par la connectique de ces pointes.

\section{Organigrammes des programmes de mesure.}

3.1 INTRODUCTION. - Le programme de mesure «simultanée » du pouvoir thermoélectrique et de la conductivité contient quatre étapes distinctes : 
- Mesures de la température du porte-échantillon: $T_{\text {ech }}(J)$. La plaquette de polyparaphénylène ayant une faible épaisseur $(\approx 1 \mathrm{~mm})$ nous pouvons considérer que sa température est sensiblement la même que celle de son support.

Après un. cycle de mesure, noté $(J)$, la valeur de $T_{\text {ech }}(J)$ est augmentée de $5 \mathrm{~K}$ et une nouvelle détermination des caractéristiques électriques du matériau étudié est effectuée (cycle: $J+1$ ).

- Mesure de la tension d'offset $\left(\Delta V_{0}\right)$ lorsque la différence de température des deux pointes de test $\left(\Delta T_{0}\right)$ est inférieure à $1{ }^{\circ} \mathrm{C}$.

- Détermination de la valeur de la conductivité du matériau, à la température $T_{\text {ech }}(J)$, en effectuant simultanément l'injection du courant et l'acquisition de la tension qui en résulte.

- Mesure de la différence de potentiel Seebeck $\left(\Delta V_{1}\right)$. Elle est consécutive à l'application d'une différence de température au niveau des pointes de mesure de tension, telle que: $\Delta T_{1}=T_{1}-T_{0} \approx 9^{\circ} \mathrm{C}$.

Calcul du coefficient Seebeck selon la relation suivante:

$$
S=-\frac{\Delta V}{\Delta T}=-\frac{\Delta V_{1}-\Delta V_{0}}{\Delta T_{1}-\Delta T_{0}}
$$

3.2 ORganigRAMME DE MESURE. - Nous donnons sur la figure 7 uniquement l'organigramme général du programme de mesure ; des sous-programmes relatifs aux mesures de $\sigma$ et de $S$ peuvent être facilement réalisés en tenant compte des conditions de mesure que nous avons décrites précédemment.

\section{Résultats expérimentaux.}

A titre d'exemple, nous présentons les mesures obtenues dans le cas d'échantillons de polyparaphénylène PPP implantés avec des ions sodium de basse énergie $(30 \mathrm{keV})$ et de haute énergie $(250 \mathrm{keV})$, les autres paramètres d'implantation étant par ailleurs identiques : fluence $D=10^{16}$ ions $/ \mathrm{cm}^{2}$ et courant d'irradiation $j=1 \mu \mathrm{A} / \mathrm{cm}^{2}$. Les représentations sont celles initialement annoncées :

$$
\log \sigma=f\left(\frac{1}{T}\right) \text { et } S=f\left(\frac{1}{T}\right) \text { (Figs. } 8 \text { et 9), puis } \log \sigma \sqrt{T}=f\left(T^{-1 / 4}\right) \text { et } \frac{S}{T}=f\left(T^{-1 / 4}\right)
$$

(Figs. 10 et 11); elles complètent des résultats antérieurs [13] relatifs à la seule étude de $\log \sigma$ en fonction de la température à partir de laquelle les énergies d'activation ont été évaluées. Nous devons immédiatement noter que le signe de $S$ dépend de l'énergie d'implantation, ce qu'une étude systématique précédente $[9,14]$ nous avait déjà montré : alors que pour les basses énergies on obtient une semi-conduction de type $n$ avec les alcalins, aux hautes énergies on obtient une semi-conduction de type $\mathrm{p}$, et ce en fait quel que soit le type d'ions implantés. Par ailleurs, on doit noter l'évolution totalement différente de $\log \sigma$ et de $S$ en fonction de $1 / T$ selon que l'on considère l'implantation à basse énergie (Fig. 8) ou l'implantation à haute énergie (Fig. 9) : alors que dans ce dernier cas seul un processus de saut à distance variable apparaît (représentations en fonction de $T^{-1 / 4}$ de la figure 11 strictement linéaires avec $S$ petit), on doit noter l'apparition de différents mécanismes de conduction lors de l'implantation aux plus basses énergies : pour $1000 / T \approx 3,2$ (soit $T \approx 312 \mathrm{~K}$ ) on note un changement de pente (Fig. 8a) de la représentation $\log \sigma=f(1 / T)$ alors que la courbe (Fig. 8 b) $S=f(1 / T)$ présente un phénomène de transition pouvant être attribué soit au passage (cf. par exemple la Fig. 6-39 de la Réf. [5]) d'une conduction dans les états localisés à 


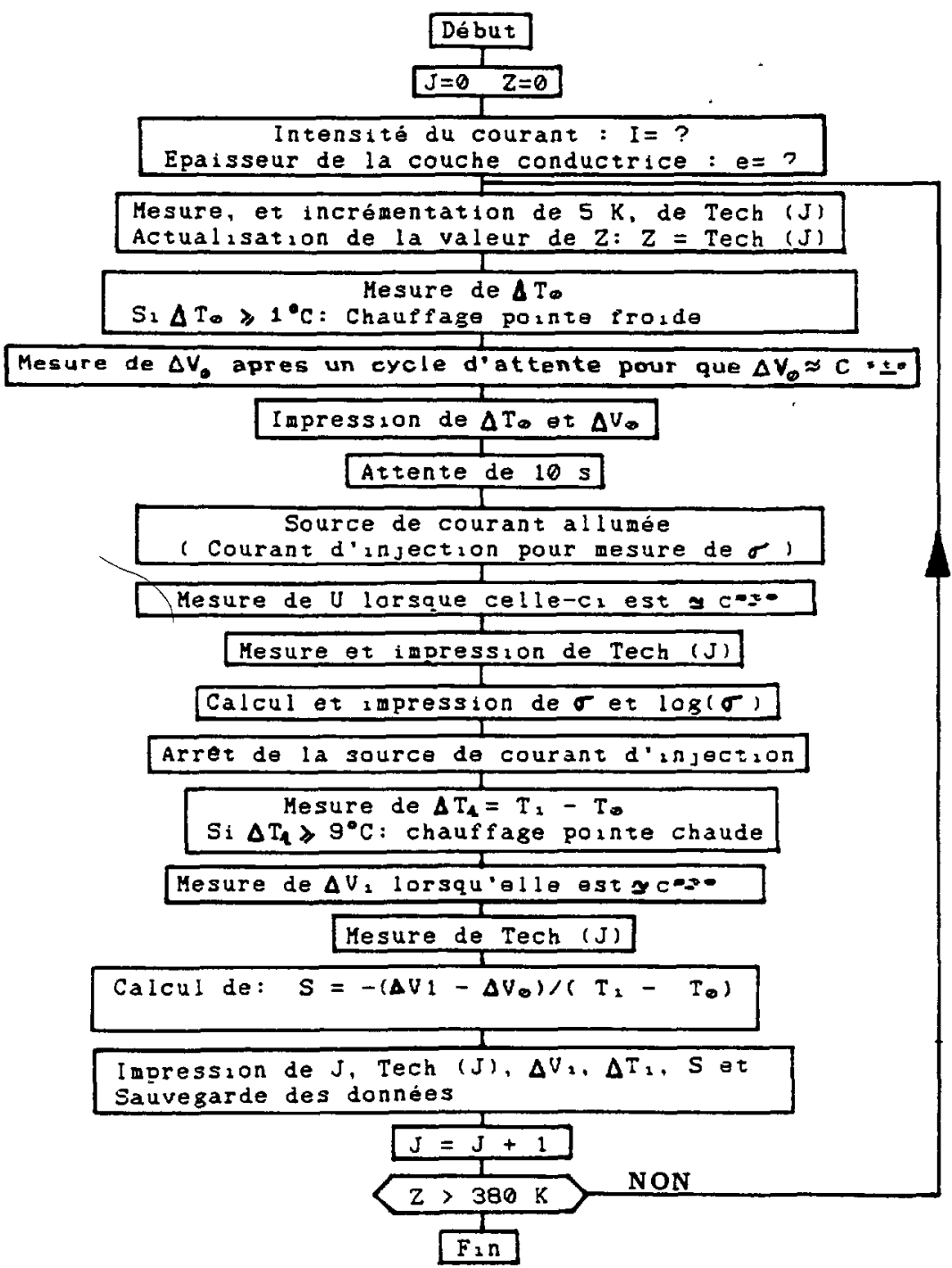

Fig. 7. - Organigramme de mesure simultanée de $(\sigma)$ et de $(S)$.

[Flow chart of simultaneous measures of $(\sigma)$ and $(S)$.]

une conduction dans les états étendus, soit au passage d'une conduction de type saut à distance variable (aux plus basses températures) à une conduction dans les états localisés au voisinage des queues de bande. Compte tenu de la faible évolution de $S$ aux basses températures, il semblerait cependant que cette deuxième interprétation soit la plus acceptable. (En effet, dans le cas de la première interprétation, le rapport de la pente de $\log \sigma=f\left(\frac{1}{T}\right)$ sur la pente de $S=f\left(\frac{1}{T}\right)$ devrait être égal à

$$
r=\frac{E_{\mathrm{A}}-E_{\mathrm{F}}+\Delta w_{1}}{\frac{k}{q}\left(E_{\mathrm{A}}-E_{\mathrm{F}}\right)}
$$




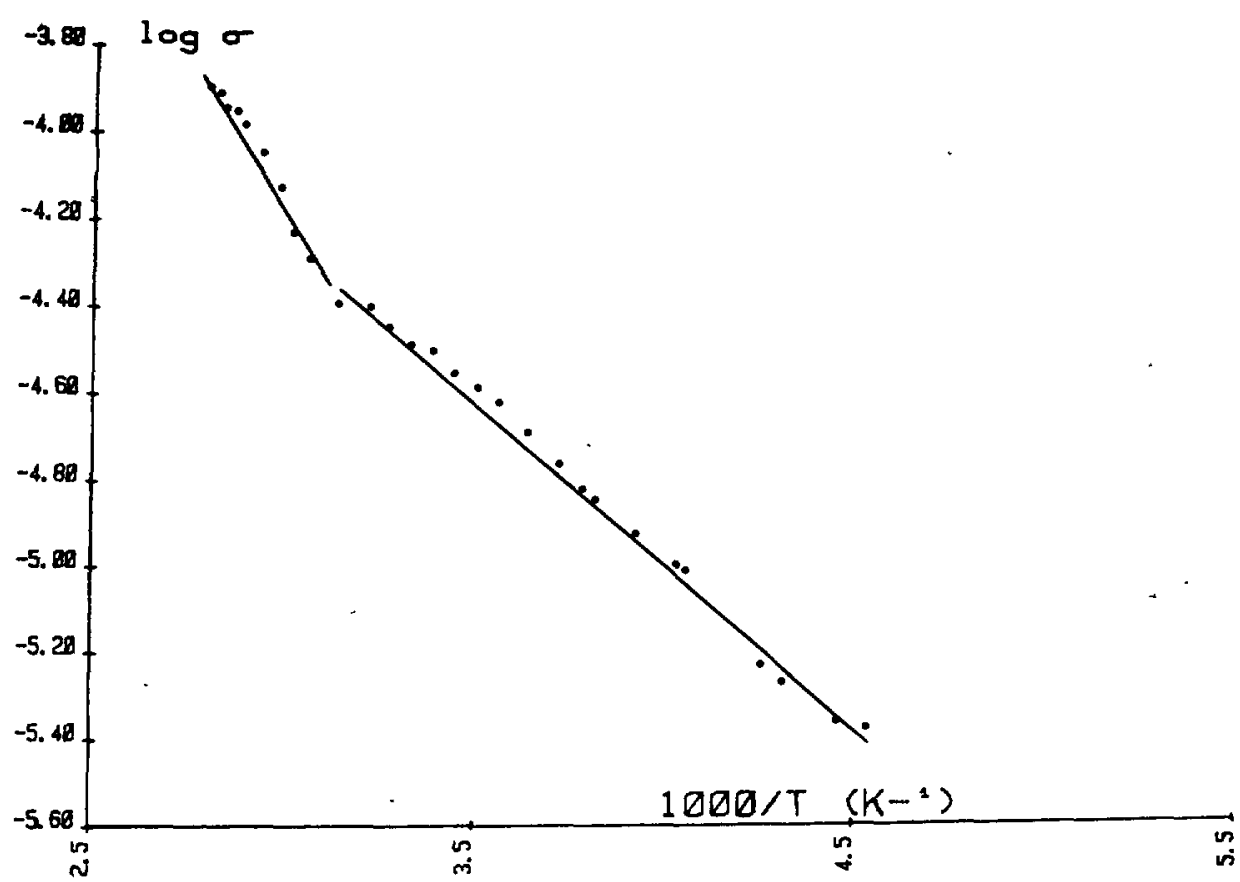

Fig. 8a. - $\log \sigma=f(1 / T)$ pour un échantillon de PPP dopé en ions $\mathrm{Na}^{+}$à basse énergie $\left(E=30 \mathrm{keV}, j=1 \mu \mathrm{A} / \mathrm{cm}^{2}, D=10^{16}\right.$ ions $\left./ \mathrm{cm}^{2}\right)$.

$\left[\log \sigma=f(1 / T)\right.$ for $\mathrm{Na}$ implanted polyparaphenylene sample $\left(E=30 \mathrm{keV}, j=1 \mu \mathrm{A} / \mathrm{cm}^{2}\right.$, $D=10^{16}$ ions $\left./ \mathrm{cm}^{2}\right)$.]

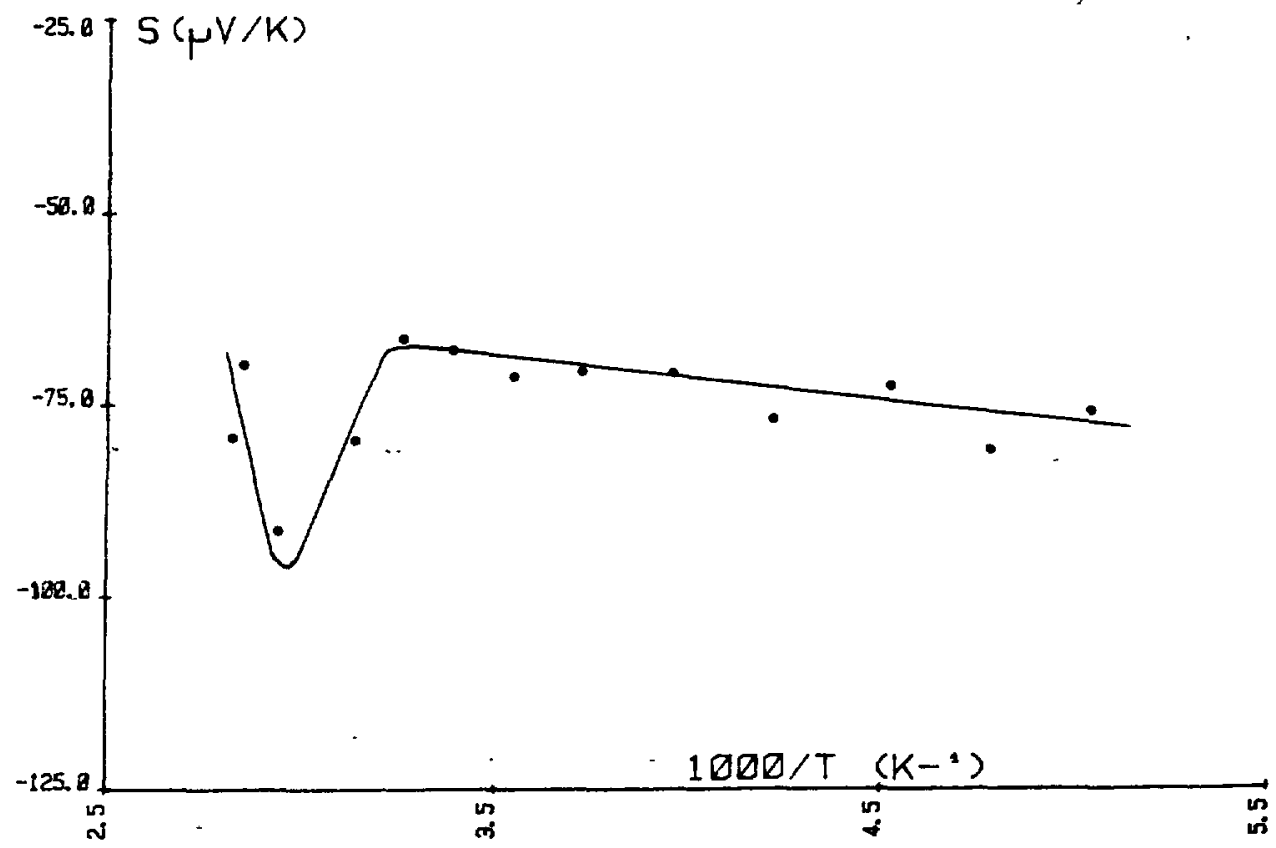

Fig. 8 b. $-S=f(1 / T)$ pour un échantillon de PPP dopé en ions $\mathrm{Na}^{+}\left(E=30 \mathrm{keV}, j=1 \mu \mathrm{A} / \mathrm{cm}^{2}\right.$, $D=10^{16}$ ions $/ \mathrm{cm}^{2}$ ).

$\left[S=f(1 / T)\right.$ for Na implanted PPP sample $\left(E=30 \mathrm{keV}, j=1 \mu \mathrm{A} / \mathrm{cm}^{2}, D=10^{16}\right.$ ions $\left./ \mathrm{cm}^{2}\right)$. 


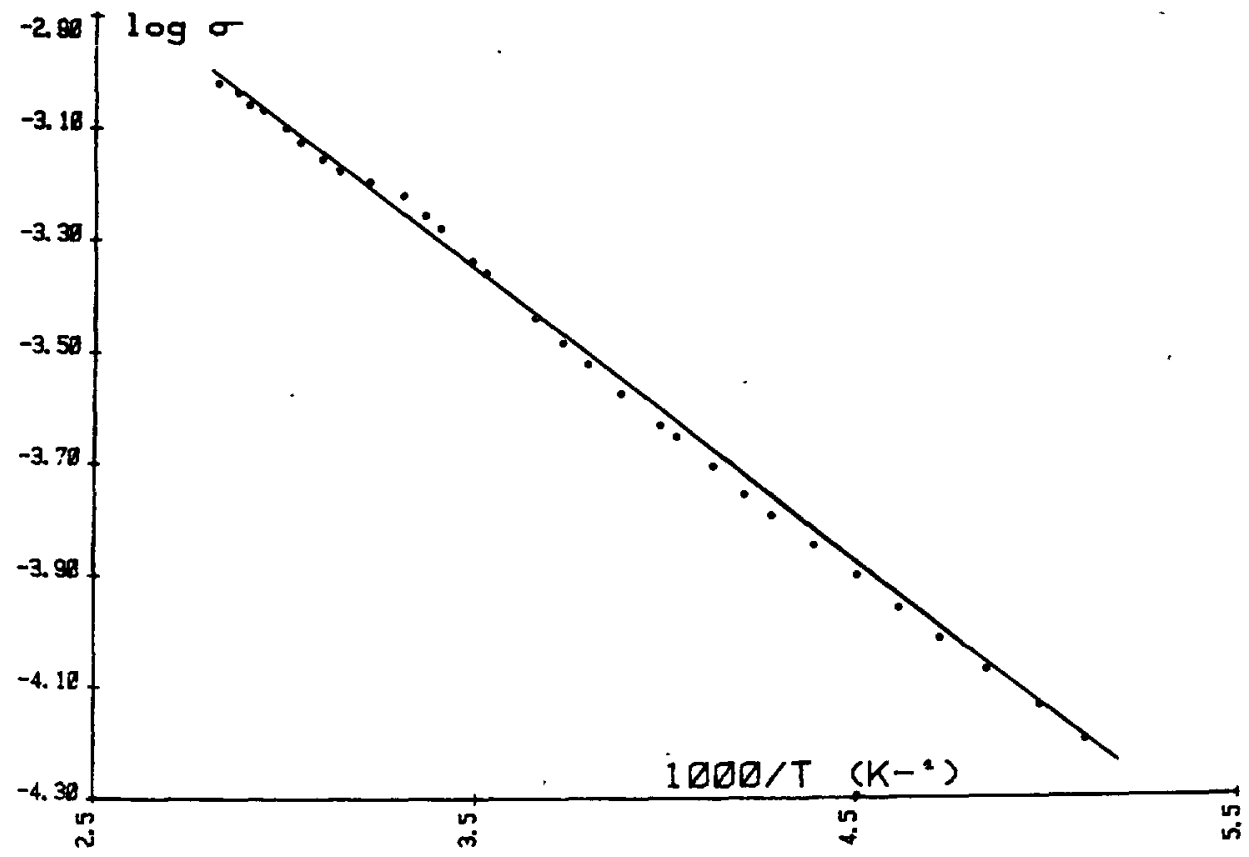

Fig. 9a. - $\log \sigma=f(1 / T)$ pour un échantillon de PPP implanté avec des ions $\mathrm{Na}^{+}$à haute énergie $\left(E=250 \mathrm{keV}, j=1 \mu \mathrm{A} / \mathrm{cm}^{2}, D=10^{16}\right.$ ions $\left./ \mathrm{cm}^{2}\right)$.

$\left[\log \sigma=f(1 / T)\right.$ for $\mathrm{Na}$ implanted polyparaphenylene sample $\left(E=250 \mathrm{keV}, j=1 \mu \mathrm{A} / \mathrm{cm}^{2}\right.$, $D=10^{16}$ ions $\left./ \mathrm{cm}^{2}\right)$.]

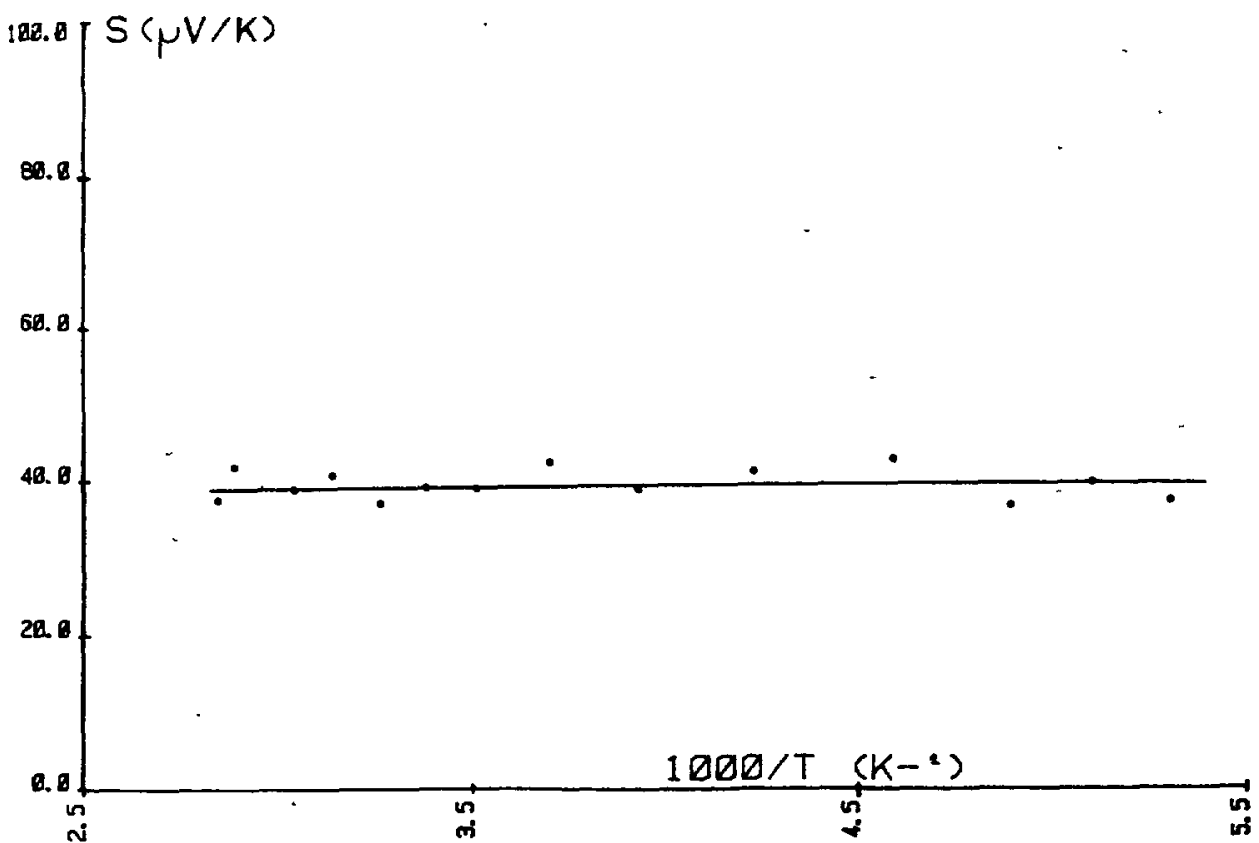

Fig. 9b. $-S=f(1 / T)$ pour un échantillon de PPP implanté avec des ions $\mathrm{Na}^{+}(E=250 \mathrm{keV}$, $j=1 \mu \mathrm{A} / \mathrm{cm}^{2}, D=10^{16}$ ions $\left./ \mathrm{cm}^{2}\right)$.

$\left[S=f(1 / T)\right.$ for $\mathrm{Na}$ implanted polyparaphenylene sample $\left(E=250 \mathrm{keV}, \quad j=1 \mu \mathrm{A} / \mathrm{cm}^{2}\right.$, $D=10^{\text {io }}$ ions $\left(\mathrm{cm}^{2}\right)$.] 


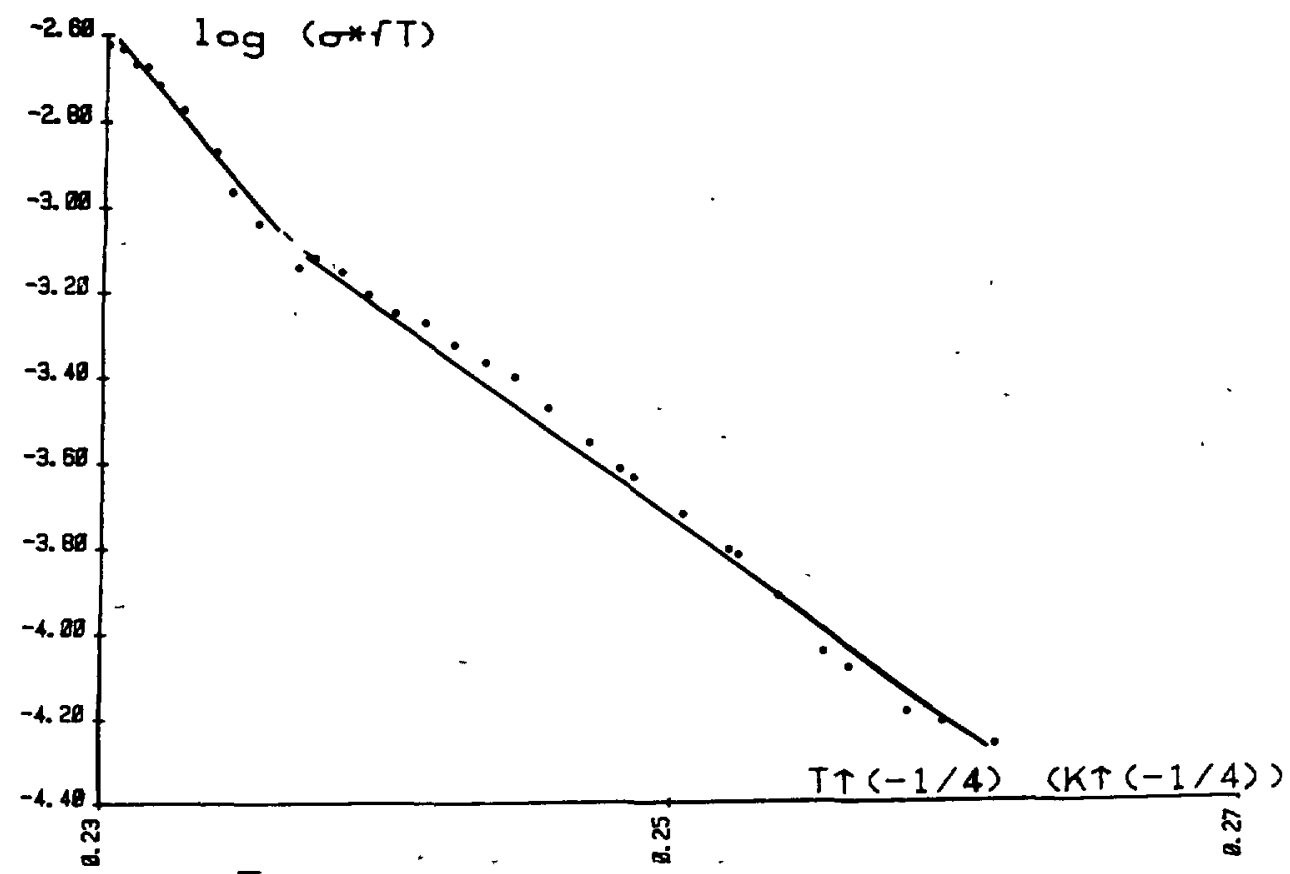

Fig. 10a. $-\log (\sigma \sqrt{T})=f\left(T^{-1 / 4}\right)$ pour un échantillon de PPP dopé en ions $\mathrm{Na}^{+}$à basse énergie $\left(E=30 \mathrm{keV}, j=1 \mu \mathrm{A} / \mathrm{cm}^{2}, D=10^{16}\right.$ ions $\left./ \mathrm{cm}^{2}\right)$.

$\left[\log (\sigma \sqrt{T})=f\left(T^{-1 / 4}\right)\right.$ for $\mathrm{Na} \quad$ implanted PPP sample $\left(E=30 \mathrm{keV}, \quad j=1 \mu \mathrm{A} / \mathrm{cm}^{2}\right.$, $D=10^{16}$ ions $\left./ \mathrm{cm}^{2}\right)$.]

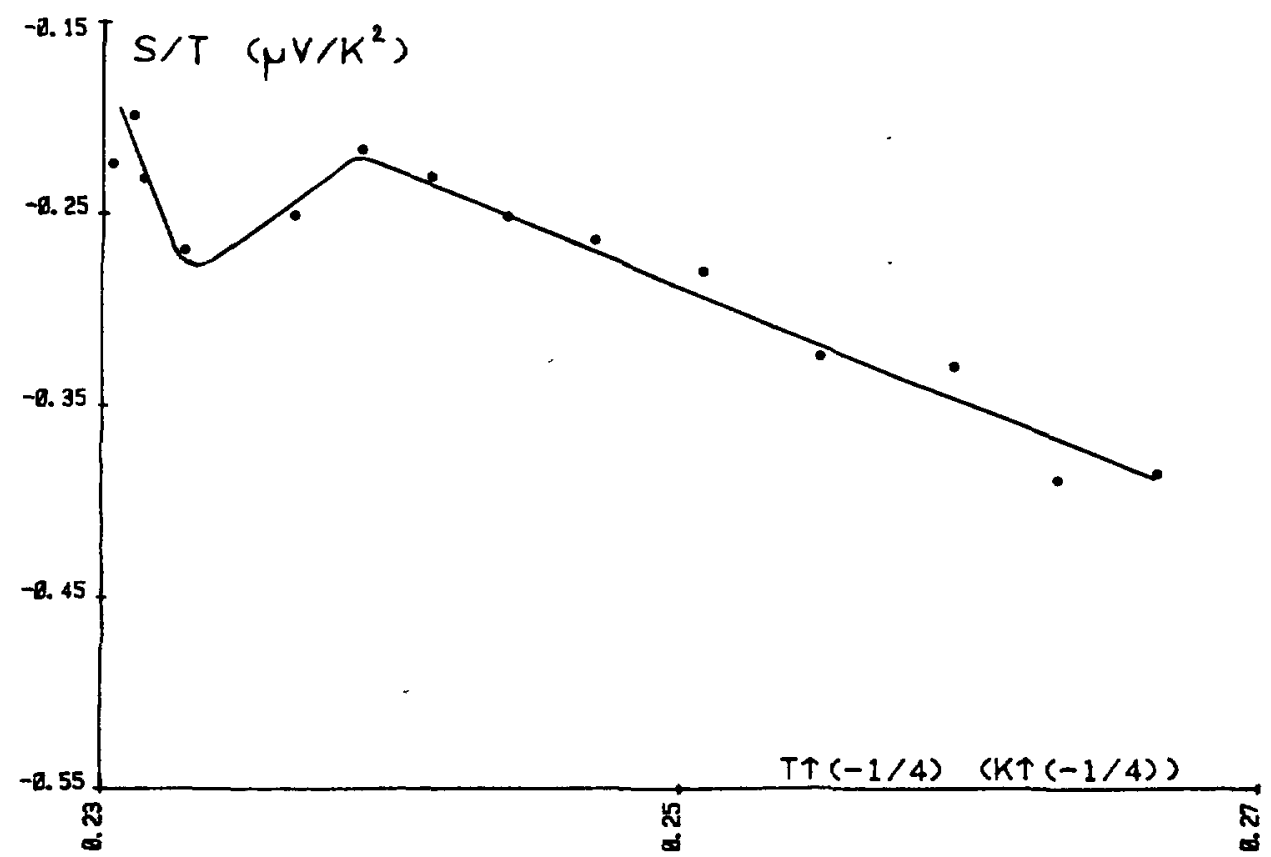

Fig. 10b. $-S / T=f\left(T^{-1 / 4}\right)$ pour un échantillon de PPP dopé en ions $\mathrm{Na}^{+}\left(E=30 \mathrm{keV}, j=1 \mu \mathrm{A} / \mathrm{cm}^{2}\right.$, $D=10^{16}$ ions $/ \mathrm{cm}^{2}$ ).

$\left[S / T=f\left(T^{-1 / 4}\right)\right.$ for Na implanted PPP sample $\left(E=30 \mathrm{keV}, j=1 \mu \mathrm{A} / \mathrm{cm}^{2}, D=10^{16} \mathrm{ions} / \mathrm{cm}^{2}\right)$. $]$ 
$\left(E_{\mathrm{A}}-E_{\mathrm{F}}\right)$ traduit l'écart d'énergie entre les niveaux localisés et le niveau de Fermi, $\Delta w_{1}$ est l'énergie séparant deux niveaux localisés et $\frac{k}{q}=8,61 \times 10^{-5} \mathrm{~V} / \mathrm{K}$; avec $\Delta w_{1} \ll\left(E_{\mathrm{A}}-E_{\mathrm{F}}\right)$ ce rapport théorique $r$ est de l'ordre de $10^{4} \mathrm{~K} / \mathrm{V}$ alors qu'expérimentalement il est trouvé de l'ordre de $\left.10^{5} \mathrm{~K} / \mathrm{V}\right)$. On peut signaler que des formes analogues de transition pour $S=f(T)$ ont pu être observées $[15,16]$ : dans le cas des semi-conducteurs amorphes [16] elles ont été attribuées à la présence d'oxygène qui introduit un deuxième type de porteurs. Cette interprétation ne peut pas être retenue dans notre étude car cette transition devrait être aussi observée dans le cas des implantations à plus haute énergie (ce qui n'est pas le cas : Figs. 9 et 11) qui conduisent à des concentrations en oxygène (contrôlées par Sims [2]) voisines de celles obtenues aux basses énergies.

A partir des courbes $\frac{S}{T}=f\left(T^{-1 / 4}\right)$ exploitées soit dans le domaine des basses températures (Fig. 10b), où a lieu le mécanisme de saut à distance variable, soit dans tout le domaine de température (Fig. 11b) lorsque seul ce mécanisme intervient, on peut déterminer le paramètre $\gamma=\frac{1}{N\left(E_{\mathrm{F}}\right)}\left[\frac{\mathrm{d} N(E)}{\mathrm{d} E}\right]_{E_{\mathrm{F}}}$ caractéristique d'une évolution linéaire de la fonction densité d'états au voisinage du niveau de Fermi ; selon un calcul développé par Nagels [17] ce paramètre $\gamma$ est tel que $\frac{S}{T}=-\frac{\pi^{2} k^{2}}{12 q} \gamma\left(\frac{T_{0}}{T}\right)^{1 / 4}$

Pour l'échantillon implanté à basse énergie (courbe $8 \mathrm{~b}$ ) et de type $n$ on obtient $\gamma \approx 14 \mathrm{eV}^{-1}$, alors que pour l'échantillon implanté à haute énergie (courbe $9 \mathrm{~b}$ ) et de type $\mathrm{p}$

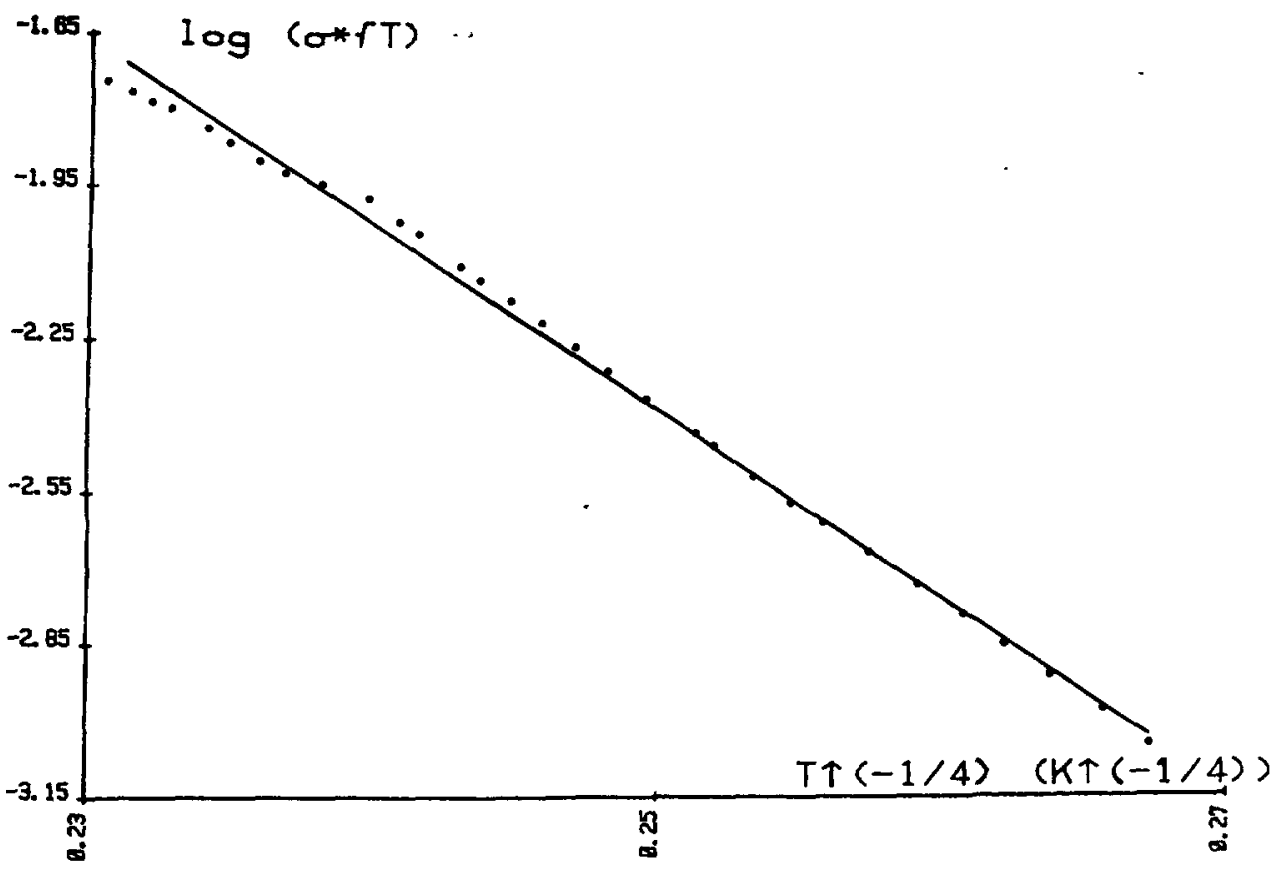

Fig. 11a. - $\log (\sigma \sqrt{T})=f\left(T^{-1 / 4}\right)$ lors d'une implantation de l'échantillon de PPP en ions $\mathrm{Na}^{+}$à haute énergie $\left(E=250 \mathrm{keV}, j=1 \mu \mathrm{A} / \mathrm{cm}^{2}, D=10^{16} \mathrm{ions} / \mathrm{cm}^{2}\right)$.

$\left[\log (\sigma \sqrt{T})=f\left(T^{-1 / 4}\right)\right.$ for $\mathrm{Na}$ implanted PPP sample $\left(E=250 \mathrm{keV}, \quad j=1 \mu \mathrm{A} / \mathrm{cm}^{2}\right.$, $D=10^{16}$ ions $\left./ \mathrm{cm}^{2}\right)$.] 


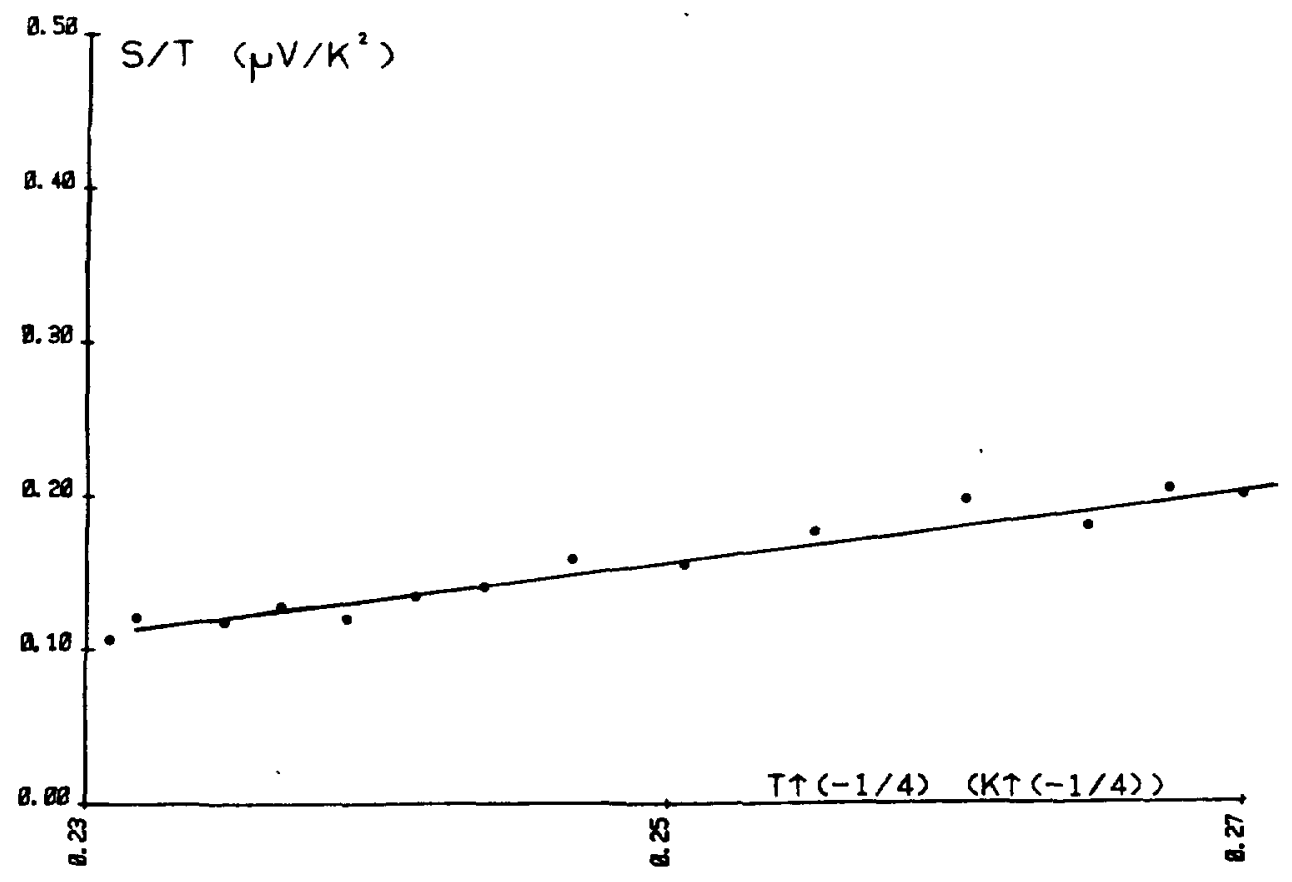

Fig. 11b. $-S / T=f\left(T^{-1 / 4}\right)$ lors d'une implantation de l'échantillon de PPP en ions $\mathrm{Na}^{+}(E=250 \mathrm{keV}$, $j=1 \mu \mathrm{A} / \mathrm{cm}^{2}, D=10^{16}$ ions $/ \mathrm{cm}^{2}$ ).

$\left[S / T=f\left(T^{-1 / 4}\right)\right.$ for $\mathrm{Na}$ implanted PPP sample $\left(E=250 \mathrm{keV}, j=1 \mu \mathrm{A} / \mathrm{cm}^{2}, D=10^{16}\right.$ ions $\left./ \mathrm{cm}^{2}\right)$.]

on trouve $\gamma \approx-5 \mathrm{eV}^{-1}$ : on a pu vérifier expérimentalement que le signe de $\gamma$ était de façon générale toujours positif dans le cas de couches dopées $n$ et toujours négatif - dans le cas des couches dopées $\mathrm{p}$, en accord avec le schéma habituel décrivant la bande de défauts localisés au voisinage du niveau de Fermi (cf. par exemple la Fig. 2-16b de la Réf. [5]).

\section{Conclusion.}

Nous avons développé un banc automatisé de mesure du pouvoir thermoélectrique et de la conductivité : il permet en particulier d'étudier ces paramètres en fonction de la température dans le cas de couches implantées relativement fragiles. L'application de la théorie des semiconducteurs amorphes au cas de polymères électroactifs implantés nous permet alors de montrer de façon irréfutable le rôle de l'énergie d'implantation : pour de fortes énergies seuls des défauts sont générés conduisant à une semi-conduction de type $\mathrm{p}$ et à un mécanisme de saut à distance variable alors que pour les implantations à basse énergie un mécanisme de conduction dans les états générés par le dopage est mis en évidence par la mesure simultanée de la conductivité et du pouvoir thermoélectrique.

\section{Bibliographie}

[1] Duroux J. L., Moliton A. et Froyer G., Nucl. Inst Meth. B 34 (1988) 450.

[2] Ratier B., Thèse d'Université de Limoges, n 36 (1990). 
[3] Bredas J. L., Chance R. R. et Silbey R., Phys. Rev. B 26 (1982) 5843.

[4] Nagels P., dans Amorphous Semiconductors, M. H. Brodsky Ed., Topics in Applied Physics, vol. 36, Springer Verlag, Berlin (1985).

[5] Mott N. F. et Davis E. A., Electronic processes in non crystalline materials (Clarendon Press, Oxford, 1979) p. 235-236.

[6] Chaikin P. M. et Kwak J. F., Rev. Sci. Instrum 42 (1975) 1975.

[7] BRAU A., Thèse d'état, Nice (1976).

[8] Goldsmid H. J., J. Phys. E: Sci. Instrum. 19 (1986) 921.

[9] Moliton A., Ratier B., Guille B. et Froyer G., Mol. Cryst. Liq. Cryst. 186 (1990) 223.

[10] Nagels P., Tichy L. et Ticha M., J. non Cryst. Sol. 59, 60 (1983) 1015.

[11] Heikes R. R. et URe R. W., Thermoelectricity - Intersciences Publishers, New York (1961) p. 313.

[12] Legros R., Les Semiconducteurs - Eyrolles, Paris (1974) p. 207.

[13] Moliton A., Duroux J. L., Ratier B. et Froyer G., J. Polym. Sci., Phys. Ed. 28 (1990) 17.

[14] Moliton A. et Duroux J. L.; Bull. Off. Ppté Indus. 53 (1987) 600.

[15] von Molnar et Holtzberg, AIP Conf. Proc. 10 (1972) 1259.

[16] BeYer W. et StUKe J., Amorphous and Liquid Semiconductors, Taylor and Francis, London (1974) p. 251.

[17] Nagels P., Rotti M. et Gevers R., J. non Cryst. Sol. 59, 60 (1983) 65. 